\title{
Difficulties in interpretation of oral food challenge results
}

\author{
Julia Gawryjołek ${ }^{1}$, Alicja Wycech², Adrianna Smyk², Aneta Krogulska ${ }^{1}$
}

${ }^{1}$ Department of Paediatrics, Allergology and Gastroenterology, Ludwik Rydygier Collegium Medicum in Bydgoszcz, Nicolaus Copernicus University in Torun, Poland

${ }^{2}$ Student Research Cirle, Paediatric Allergology and Gastroenterology, Ludwik Rydygier Collegium Medicum in Bydgoszcz, Nicolaus Copernicus University in Torun, Poland

Adv Dermatol Allergol 2021; XXXVIII (5): 721-726 DOI: https://doi.org/10.5114/ada.2021.106248

\begin{abstract}
Oral food challenge is the gold standard in diagnosing food allergies; however, many testing protocols are available. The present article illustrates the difficulties associated with interpreting oral challenge tests with the example of a six-year-old boy with allergy to hen's eggs. The symptoms observed on the first day of challenge indicated a negative result; however, the consumption of the cumulative dose resulted in anaphylaxis. The interpretation of the oral food challenge can be complicated. The criteria used to determine a positive or negative result are sometimes ambiguous. An accurate interpretation of the results is key to determining correct management in children with food allergy.
\end{abstract}

Key words: oral food challenge, egg allergy, baked egg, child, food allergy, diagnosis.

\section{Introduction}

Oral food challenge (OFC) plays a crucial role in the diagnosis of food allergy. Although history, skin prick testing (SPT), and serum specific IgE (slgE) tests can also be indicative of a food allergy (FA), they do not allow clear confirmation or exclusion. For patients with suspected FA, especially growing children, it is essential to establish the correct diagnosis: a false-negative OFC result exposes the patient to an anaphylactic reaction, and a false-positive result can result in nutritional deficiencies, developmental disorders and impaired quality of life, and even acute allergic reactions.

One of the most common forms of FA is hen's egg allergy, which is estimated to affect $0.5-2.5 \%$ of children; it is also one of the most common causes of anaphylaxis, especially in infants and young children [1, 2]. The condition is most commonly diagnosed during early childhood: according to the EuroPrevall survey, the symptoms become apparent at a mean age of 12 months, with a diagnosis occurring at 17 months, and symptoms typically appear after the first documented contact with eggs [3]. Hen's egg allergy often co-occurs with other FA e.g. peanut allergy, and other atopic diseases, such as atopic dermatitis (AD), allergic rhinitis (AR) or asthma; Xepapadaki et al. report that $70 \%$ of children with hen's egg allergy also demonstrate $\mathrm{AD}$ [3]. The time to acquiring tolerance to egg allergens varies. It has been found that $68 \%$ of children acquire tolerance to cooked hen's eggs by the age of 16 years old [4], while Peters et al. note that half of children develop tolerance around the age of 2 years [5]. Interestingly, patients with IgE-dependent FA are more likely to demonstrate more persistent egg allergy, similar to other forms of FA [2].

Clinically, three groups of patients can be distinguished: those who are sensitive to hen's eggs, but tolerate them in both raw and heat-treated, or "baked", forms; those who are allergic to raw eggs, but tolerate heat-treated ones; and those with an allergy to hen's eggs in either form. The primary allergens identified in hen's eggs are ovomucoid (Gal d 1), ovalbumin (Gal d 2), ovotransferrin (Gal d 3), lysozyme (Gal d 4) and livetin (Gal d 5). Of these, ovomucoid is considered the most allergenic, mainly due to its thermostability and resistance to digestion by enzymes [6].

A diagnosis of egg allergy is based on a standard procedure comprising a detailed medical history, physical examination, sensitization assessment, i.e. SPT and/ or slgE, and OFC [7]. Although the presence of a larger wheal in the SPT and a higher level of slgE in the blood indicate a higher risk of the allergic reaction, they do not allow its severity to be determined. It has been found that egg protein slgE levels $\geq 7 \mathrm{kU} / \mathrm{l}$ (or $\geq 2 \mathrm{kU} / \mathrm{l}$ for chil-

Address for correspondence: Julia Gawryjołek MD, Department of Paediatrics, Allergology and Gastroenterology, Ludwik Rydygier Collegium Medicum, Nicolaus Copernicus University, 9 M. Skłodowskiej-Curie St, 85-094 Bydgoszcz, Poland, phone: +48 525854850 , fax: +48 5258540 86, e-mail: gawryjolek.j@gmail.com Received: 8.09.2020, accepted: 2.02.2021. 
dren $<2$ years of age) and SPT $\geq 7 \mathrm{~mm}$ suggest a 95\% likelihood of obtaining a positive OFC. In addition, egg protein $\mathrm{slgE} \leq 2 \mathrm{kU} / \mathrm{l}$ and $\mathrm{SPT} \leq 3 \mathrm{~mm}$ indicate a $50 \%$ likelihood of obtaining a negative OFC [8]. Including component resolved diagnostics (CRD) in the diagnostic process can help differentiate sensitivity from allergy and allow the tolerance to raw and heat-treated eggs to be estimated [6]. It has been found that the level of Gal d 1 is a good marker of heat-treated egg sensitivity, and that high levels are associated with persistent egg allergy [9].

Table 1. Evaluation of positive and negative likelihood ratio of OFC with baked egg based on cut-off levels for slgE and SPT with egg [10-18]

\begin{tabular}{|c|c|c|c|}
\hline Cut-off & Positive LR & Negative LR & Reference \\
\hline \multicolumn{4}{|l|}{ OM slgE: } \\
\hline$>10.8$ & 13.75 & 0.47 & [10] \\
\hline$>1.16$ & 2.06 & 0.06 & [10] \\
\hline$>12.8$ & 5.6 & 0.76 & [11] \\
\hline$<8$ & 1.63 & 0.85 & [12] \\
\hline$>9.74$ & 7 & 0.94 & [13] \\
\hline \multicolumn{4}{|l|}{ EW slgE: } \\
\hline$>30.7$ & 10.5 & 0.6 & {$[10]$} \\
\hline$>10$ & 3.33 & 0.85 & {$[14]$} \\
\hline$>26.2$ & 2.4 & 0.93 & {$[11]$} \\
\hline$>0.78$ & 1.48 & 0.11 & {$[11]$} \\
\hline$>9.65$ & 7.4 & 0.66 & [13] \\
\hline$<8$ & 2.40 & 0.53 & {$[12]$} \\
\hline \multicolumn{4}{|l|}{ EW SPT: } \\
\hline$>3$ & 1.2 & 0 & [13] \\
\hline$>11$ & 1.57 & 0.55 & {$[13]$} \\
\hline$>11$ & - & 1.01 & [15] \\
\hline$>12$ & 8 & 0.86 & [16] \\
\hline$<10$ & 3.27 & 0.36 & {$[12]$} \\
\hline \multicolumn{4}{|l|}{ OM SPT: } \\
\hline$>11$ & - & 0.82 & {$[17]$} \\
\hline \multicolumn{4}{|c|}{ Baked egg SPT: } \\
\hline$<2$ & 1.16 & 0.24 & {$[17]$} \\
\hline \multicolumn{4}{|c|}{ Raw egg SPT: } \\
\hline$>25$ & 5 & 0.96 & [16] \\
\hline \multicolumn{4}{|c|}{$\begin{array}{l}E W \text { - egg white, } L R \text { - likelihood ratio, OM - ovomucoid, slgE - specific IgE. } \\
\text { A positive } L R \text { of }>10 \text { indicates that the test result has a large effect on increas- } \\
\text { ing the probability of reacting (positive challenge), a positive } L R \text { of } 5-10 \text { indi- } \\
\text { cates that the test result has a moderate effect on increasing the probability } \\
\text { of a reaction, and a positive } L R \text { of }<5 \text { indicates a small effect on increasing } \\
\text { the probability of reacting. A negative } L R \text { of }<0.1 \text { indicates that the test result } \\
\text { is very likely to result in tolerance (i.e., passed challenge, negative challenge), } \\
\text { a negative } L R \text { of } 0.1-0.5 \text { indicates a moderate probability of passing the chal- } \\
\text { lenge, and an } L R \text { of }>0.5 \text { indicates a small effect on decreasing disease prob- } \\
\text { ability. Based on the data from Bartinikas et al. [13], an EW SPT wheal of } \\
<3 \mathrm{~mm} \text { would be highly predictive for passing a baked egg challenge. }\end{array}$} \\
\hline
\end{tabular}

Ando et al. propose $7.4 \mathrm{kU}(\mathrm{A}) / \mathrm{l}$ as a positive decision cutoff point for diagnosis of raw egg white allergy, assuming $95 \%$ specificity, and $0.6 \mathrm{kU}(\mathrm{A}) / \mathrm{l}$ as the negative point, assuming $95 \%$ clinical sensitivity; for heated egg white, the positive decision point was $10.8 \mathrm{kU}(\mathrm{A}) / \mathrm{l}$, and the negative $1.2 \mathrm{kU}(\mathrm{A}) / \mathrm{l}[10]$.

The slgE and SPT cut-off points for raw eggs are different for those associated with heat-treated eggs (Table 1) [10-18]. Lemon-Mule et al. propose that a concentration of Ovomucoid $(\mathrm{OM}) \mathrm{slgE}>50 \mathrm{kU}(\mathrm{A}) / \mathrm{l}$ indicates $>90 \%$ positive predictive value (PPV) of a positive OFC with baked egg, while egg white (EW) SPT $>15 \mathrm{~mm}$ corresponds to 60\% PPV, and EW SPT < $3 \mathrm{~mm}$ to < 5\% PPV [19].

However, CRD cannot replace OFC as a reliable diagnostic procedure [6], and OFC remains the gold diagnostic standard. Even so, despite its undoubted advantages, even the optimal OFC method, i.e. DBPCFC, has a number of disadvantages, including the possibility of anaphylaxis and difficulties in interpreting the results.

It has also been noted that correct interpretation of the results of the challenge has a significant influence on setting the correct diagnosis and implementing correct management. The present study describes the performance of OFC in a child with an egg allergy and the difficulties in interpreting the results. It also emphasises the need for correct interpretation of OFC results in the process of diagnosing FA.

\section{Case report}

A 6-year-old patient, TT, with FA to egg, as well as $A D, A R$ and conjunctivitis, was referred to the hospital for OFC with boiled hen's egg. The family did not report any history of allergy. He was breastfed until the age of 12 months. After 6 months of age, the diet was supplemented with egg yolk with good tolerance. At 12 months, immediately after consuming a few teaspoons of scrambled eggs, facial erythema and swelling appeared; this was the child's first exposure to egg white. The symptoms resolved themselves without assistance. From that time, the mother removed eggs from the child's diet.

The mother reported the case to an allergologist when the child was 18 months old. SlgE assessment indicated allergy to egg (egg white $-3 \mathrm{kU} / \mathrm{l}$, egg yolk $-0.7 \mathrm{kU} / \mathrm{l}$ ) and grass (4.5 kU/l). IgE-dependent egg allergy was diagnosed and an egg-elimination diet was recommended. From 2 years of age, hives were observed a number of times on the child's skin after contact with raw egg. At 3 years of age the mother introduced baked hen's eggs into the boy's diet, which was tolerated well. At 4 years of age, a related slgE test found an increase in the antibody level (slgE level was $24.2 \mathrm{lU} / \mathrm{ml}$ for egg white and $17.4 \mathrm{kU} / \mathrm{l}$ for yolk).

From the age of 2 years, the mother periodically reported: itchy erythema, rashes after contact with grasses, as well as blockage of the nose, watery discharge from the nose, and red and itchy eyes. 


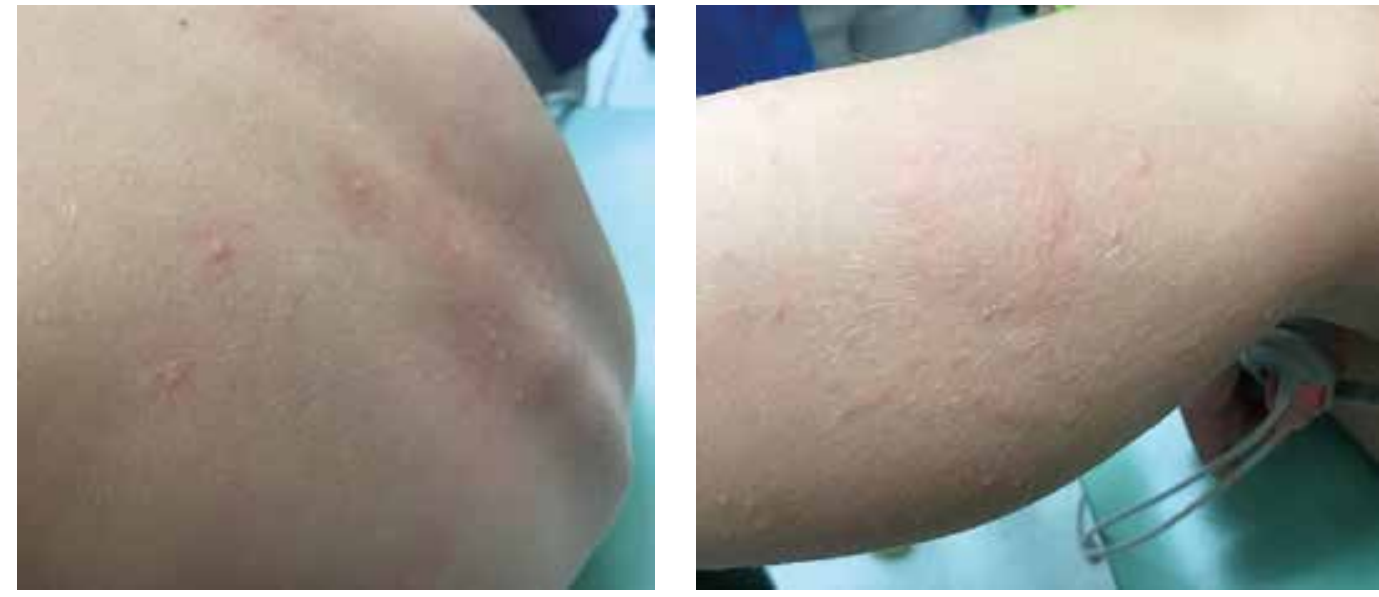

Figure 1. The skin after a cumulative dose of egg on the second day of OFC

At 6 years of age the patient was admitted to the hospital for diagnosis. SPTs were performed with egg allergens. Commercial egg white solution produced a wheal of $10 \mathrm{~mm}$ in diameter, and egg yolks of $5 \mathrm{~mm}$. Regarding the native allergens: raw egg white produced $10 \mathrm{~mm}$ wheals, yolk $4 \mathrm{~mm}$, boiled egg white $2 \mathrm{~mm}$, and cooked yolk $0 \mathrm{~mm}$ (positive control was $3 \mathrm{~mm}$, negative control $0 \mathrm{~mm}$ ). SlgE levels were found to be $0.75 \mathrm{kU} / \mathrm{l}$ for egg white, < $0.15 \mathrm{kU} / \mathrm{l}$ for egg yolk, $2.2 \mathrm{kU} / \mathrm{l}$ for Gal d 1, $1.0 \mathrm{kU} / \mathrm{l}$ for Gal d 2, and < $0.15 \mathrm{kU} / \mathrm{l}$ for Gal d 4 and conalbumin. The ISAC test showed the presence of Gal $d 1$ at a concentration of 1.3 ISU-E; and Gal d 2, Gal d 3, Gal d 4 and Gal d 5 below 0.3 ISU-E. Sensitization was also found for kiwi fruit, Act d 1 (5.7 ISU-E), sesame, Ses i 1 (10 ISU-E) and grass pollen, Phl p 1 (0.9 ISU-E), as well as birch, Bet $\vee 1$ (38 ISU- E), cat hair, fel d 4 (2.6 ISU-E) and dog hair, Can f 1, Can f 2 (1.9; 2.6 ISU-E) and moulds, Alt a 1 (54 ISU-E). The fact that the child tolerated kiwi and sesame well was supported by the interview data.

On the first day of hospitalization, an OFC with boiled eggs was administered in doses (small particle - 1/16 $1 / 8-1 / 4-1 / 2$ of the boiled egg), every $15 \mathrm{~min}$. After the first dose, the boy reported an itchy tongue. No abnormalities were noted during the physical examination. After an initial refusal to take the second dose, he was further encouraged to consume it, however, a single episode of vomiting occurred immediately. After $30 \mathrm{~min}$, the same dose was repeated, this time in a fruit mousse, which the child eagerly consumed. The challenge was continued, the patient did not make any complaints and appeared symptom free.

Next day, a single cumulative dose was administered (a whole boiled egg), which the patient consumed this time without any base. After $5 \mathrm{~min}$, the child vomited and demonstrated a running nose, hoarseness, persistent sneezing and lacrimation. After a couple of minutes, the boy reported nausea and weakness and hives were ob- served on the thigh, face and back (Figure 1), as well as generalized erythema and pruritus, tachycardia 148/min and anxiety; blood pressure also dropped to $85 / 61 \mathrm{~mm} \mathrm{Hg}$. A developing anaphylactic reaction was diagnosed. Adrenaline was administered intramuscularly, resulting in an immediate improvement in the general condition and most of the symptoms; however, the itching and hives still persisted. For this reason, prednisone was administered orally at a dose of $10 \mathrm{mg}$ together with an additional dose of a second-generation antihistamine. In the course of the observation, the child's condition improved further and the symptoms completely disappeared.

IgE-dependent allergy to hen's eggs was diagnosed. The patient was discharged home and advised to eliminate unprocessed eggs, and to maintain a regular supply of foods containing baked egg. The parents were instructed about the management of an anaphylactic reaction and the boy was provided with an anti-shock kit.

\section{Discussion}

Although the described patient demonstrated a low risk of allergic reaction (slgE for egg white and Gal d $1<95 \%$ PPV, good tolerance of baked egg, only one generalized reaction experienced in 1 year) [20], OFC incurred the most severe clinical manifestation of FA i.e. an anaphylactic reaction. Although an elevated level of hen's egg slgE can be indicative of a positive diagnosis of FA, it does not allow the severity of the allergic reaction to be predicted [9]. Therefore, it is important to remember that patients with low slgE levels and lower reactivity to SPT may also experience anaphylactic symptoms during OFC, and the physician should be aware of this when performing the test. In addition, around $70 \%$ of children with an allergy to eggs tolerate them in the cooked form (i.e. baked-egg products), the remaining 30\% do not, as in the case of the patient described herein [12]. 
Table 2. Objective and subjective symptoms during food challenges [22]

\begin{tabular}{lll}
\hline Variable & Objective & Subjective \\
\hline Skin & Erythema - flushed irritability & Pruritus - itch \\
& Urticaria - nettle rash & Tingling, mouth/ \\
& Oedema - swelling & lips \\
& Eczematous rash & \\
& Cold peripheries - shut down & \\
\hline Nasal & Congestion - sniffing & Sneezing, plus \\
& Rhinorrhoea - runny nose & itchy nose \\
\hline Ophthalmic & Ophthalmia - inflamed sore & Glazed expression \\
& eyes & \\
& Runny eyes & \\
& Swollen eyes & \\
\hline Respiratory & Wheeze & Tight/ itchy throat \\
& Hoarse voice/stridor & Tightness in chest \\
& Use of accessory muscles & Dyspnoea \\
& Tachypnoea & - difficulty \\
& & breathing \\
\hline Gastric & Vomiting & Abdominal pain \\
& Diarrhoea & Nausea \\
\hline Cardiac & Tachycardia & \\
& Hypertension & \\
& Hypotension & \\
& Cyanosis & \\
\hline Behaviour & & Irritability \\
& & Drowsiness \\
& & Decreased activity \\
& & Ancomfortable \\
& & Distress \\
\hline & & \\
& & \\
& & \\
& & \\
& & \\
& & \\
& & \\
& & \\
& & \\
& & \\
& &
\end{tabular}

Despite the implementation of increasingly advanced diagnostic methods, OFC is still widely acknowledged as the best method for detecting FA. Although DBPCFC is regarded as the gold standard in the diagnosis of $A P$, its results are difficult to interpret. Many protocols exist for conducting OFC, as do a number of criteria for determining a positive or negative result. According to PRACTALL consensus, the result of DBPCFC is considered positive, and the test terminated, following the occurrence of either persistent objective symptoms, subjective symptoms at three subsequent doses, or a severe subjective symptom such as abdominal pain/nausea with discomfort lasting for $>45 \mathrm{~min}$ [21]. Interpretation may be complicated by the presence of objective and subjective symptoms, although to a greater degree by the latter. The objective and subjective symptoms observed in the course of OFC are presented in Table 2 [22].

The itching of the tongue that occurred in the described patient is a good example of a subjective symptom; although it may not have any significance, especially if it did not occur with other accompanying symptoms such as reddening of the mucous membranes of the mouth or throat, its occurrence may herald the development of an allergic reaction in the form of oral allergies, or the beginning of a systemic anaphylactic reaction. A similar situation, although more difficult, concerns itching of the skin, especially one that occurs without visible skin changes as it may indicate the beginning of an allergic reaction. Niggemann proposes this as an indication for the tester to extend the time before the next dose of the allergen by $15 \mathrm{~min}$, or to repeat the same dose [23]. Moreover, it should be remembered that subjective symptoms, especially in children, can be caused by the patient's reluctance to eat food, a dislike of the taste, or the nocebo effect, i.e. the negative attitude of the patient and an anticipation of the side effects of provocation. Subjective symptoms such as palpitations, burning tongue or abdominal discomfort are not grounds for stopping the challenge [23].

Vomiting is an objective symptom; however, the concomitant subjective reluctance or fear of food consumption on the part of the patient or guardian may be difficult to assess, and may not always warrant OFC discontinuation. Persistent and profuse vomiting is an indication for discontinuation of OFC, and a positive indicator of FA [24]. In the presented case, the patient demonstrated a reluctance to consume the second dose, and vomited after consumption. It is difficult to clearly determine whether this incident was a symptom of the allergy or whether it resulted from the reluctance of the child to eat the food. Therefore, based on the subjectivity of the child's complaints and the lack of other clinical symptoms, OFC was continued.

The question of whether to continue the testing in such circumstances in an OFC procedure is a common dilemma. On the one hand, continuing the trial may expose the patient to severe and even life-threatening clinical symptoms; on the other, finishing early may result in an incorrect diagnosis and the introduction of unnecessary dietary restrictions. Typically, OFC continues until the point where objective symptoms are seen, without the risk of stronger allergic reactions appearing [23]. Yanagida et al. report that three-level stepwise oral food challenges performed in children with an egg allergy are stopped after the first dose in $12 \%$ of cases, after the second dose in $24 \%$, and after the third and final does in 34\% [25]. OFC was halted by mild symptoms in $20 \%$ of tested children (localized urticaria, exanthema, pruritus of the skin or pharynx or oral cavity, swollen eyelid or lips, mild abdominal pain, nausea, vomiting, diarrhoea, intermittent cough, nasal congestion, sneezing, rhinorrhoea, change in activity level, lethargy), moderate ones in 77\% (generalized urticaria, exanthema, pruritus, swollen face, throat pain, moderate abdominal pain, recurrent vomiting, diarrhoea, repetitive cough, chest tightness, wheezing, pale face, mild hypotension, tachycardia, somnolence), and severe ones in $2 \%$ (cramps, abdominal pain, repetitive vomiting, persistent cough, hoarseness, "barky" cough, audible wheezing, dyspnoea, drop in saturation, hypotension, bradycardia, loss of consciousness) [25]. 
In the present case, although a negative OFC result was obtained during the first stage of the challenge, an anaphylactic reaction was observed in the next stage, after administering an egg in the form of a single cumulative dose. The occurrence of allergic symptoms on the second day of OFC is rarely reported in the literature. A study of OFC in 242 children yielded a negative result in 193; however, five of these demonstrated immediate symptoms after consuming food on the following day at home, with three children requiring medication by the parents [26]. The authors attribute this to the use of freeze-dried food, differences in the methods of food preparation between the hospital and at home, and increased release of inflammatory cell mediators due to repeated exposure to the allergen. Moreover, it seems that although FA symptoms typically demonstrate reproducibility, in rare cases, they may not appear despite the presence of allergies [26].

A study of a series of OFC tests in 936 young children found allergy symptoms to occur 2 or 3 days after testing in $1.6 \%$ of cases, i.e. after consuming the food, despite no symptoms being observed during the day of OFC [27]. Another analysis of 490 OFCs found $7 \%$ of the tested patients to have symptoms only after the consumption of a cumulative dose on the following day, with the patients presenting a range of symptoms including urticaria, angioedema, abdominal pain, nausea, erythema and contact urticaria. The authors attribute this phenomenon to the specific oral tolerance induction (SOTI) effect: the development of short-term clinical inactivity following repeated contact with the allergen during the OFC on the first day. A break for just one night followed by a cumulative dose may again elicit clinical symptoms which were not observed on the first day after OFC [28]. Paradoxically, a lack of exposure to allergens may lead to the development of sudden allergic reactions in patients who have experienced unjustified elimination of allergens [29]; this may have happened in the presented case, when the child was fed egg white without the yolk at 12 months of age.

It is worth remembering that the risk of an allergic reaction increases if, despite a negative OFC result, the tested food is not introduced into the daily diet [30]. In some patients, re-administration of food after its elimination may trigger symptoms of an allergic reaction, including a generalized reaction. Determining the lowest dose of the allergen that the patient tolerates is extremely important from the point of view of treatment, because oral exposure to an allergen at a dose tolerated by the patient may act as a form of oral immunotherapy, hastening the achievement of tolerance. Therefore, our goal for the near future is to determine the tolerance to the half egg dose, i.e. the final fractionated dose, which the patient consumed on the first day of OFC, without complaints. In the case of a negative OFC result, and the introduction of half egg doses into the child's diet, it may be possible to accelerate the acquisition of tolerance to hen's egg allergens via the SOTI effect.

\section{Conclusions}

The correct interpretation of OFC results is essential for the correct diagnosis of food allergy. However, tolerance to baked egg does not necessarily indicate that the egg is also tolerated in its unprocessed form. Low levels of ovomucoid-slgE and the absence of symptoms after fractionated allergen supply do not always indicate the absence of the egg allergy. It is advisable to perform oral challenges with fractionated doses of the allergen and a cumulative dose on the following day.

\section{Conflict of interest}

The authors declare no conflict of interest.

\section{References}

1. Sicherer SH, Sampson HA. Food allergy: a review and update on epidemiology, pathogenesis, diagnosis, prevention, and management. J Allergy Clin Immunol 2018; 141: 41-58.

2. Sicherer SH, Wood RA, Vickery BP, et al. The natural history of egg allergy in an observational cohort. J Allergy Clin Immunol 2014; 133: 492-9.

3. Xepapadaki P, Fiocchi A, Grabenhenrich L, et al. Incidence and natural history of hen's egg allergy in the first 2 years of life-the EuroPrevall birth cohort study. Allergy 2016; 71: 350-7.

4. Savage JH, Matsui EC, Skripak JM, Wood RA. The natural history of egg allergy. J Allergy Clin Immunol 2007; 120: 1413-7.

5. Peters RL, Dharmage SC, Gurrin LC, et al. The natural history and clinical predictors of egg allergy in the first 2 years of life: a prospective, population-based cohort study. J Allergy Clin Immunol 2014; 133: 485-91.

6. Matricardi PM, Kleine-Tebbe J, Hoffmann HJ, et al. EAACI molecular allergology user's guide. Pediatr Allergy Immunol 2016; 27 (Suppl 23): 1-250.

7. Muraro A, Werfel T, Hoffmann-Sommergruber K, et al. EAACl food allergy and anaphylaxis guidelines: diagnosis and management of food allergy. Allergy 2014; 69: 1008-25.

8. Nowak-Wegrzyn A, Assa'ad AH, Bahna SL, et al. Work Group report: oral food challenge testing. J Allergy Clin Immunol 2009; 123 (6 Suppl): S365-83.

9. Alessandri C, Zennaro D, Scala E, et al. Ovomucoid (Gal d 1) specific IgE detected by microarray system predict tolerability to boiled hen's egg and an increased risk to progress to multiple environmental allergen sensitisation. Clin Exp Allergy 2012; 42: 441-50.

10. Ando H, Moverare R, Kondo Y, et al. Utility of ovomucoidspecific IgE concentrations in predicting symptomatic egg allergy. J Allergy Clin Immunol 2008; 122: 583-8.

11. Caubet JC, Nowak-Wegrzyn A, Moshier E, et al. Utility of casein-specific IgE levels in predicting reactivity to baked milk. J Allergy Clin Immunol 2013; 131: 222-e4.

12. Saifi M, Swamy N, Crain M, et al. Tolerance of a high-protein baked-egg product in egg-allergic children. Ann Allergy Asthma Immunol 2016; 116: 415-9.

13. Bartnikas LM, Sheehan WJ, Larabee KS, et al. Ovomucoid is not superior to egg white testing in predicting tolerance to baked egg. J Allergy Clin Immunol Pract 2013; 1: 354-60.

14. Lieberman JA, Huang FR, Sampson HA, Nowak-Wegrzyn A. Outcomes of 100 consecutive open, baked-egg oral food 
challenges in the allergy office. J Allergy Clin Immunol 2012; 129: 1682-4.e2.

15. Peters RL, Allen KJ, Dharmage SC, et al. Skin prick test responses and allergen-specific IgE levels as predictors of peanut, egg, and sesame allergy in infants. J Allergy Clin Immunol 2013; 132: 874-80.

16. Turner PJ, Kumar K, Fox AT. Skin testing with raw egg does not predict tolerance to baked egg in egg-allergic children. Pediatr Allergy Immunol 2014; 25: 657-61.

17. Tan JW, Campbell DE, Turner PJ, et al. Baked egg food challenges - clinical utility of skin test to baked egg and ovomucoid in children with egg allergy. Clin Exp Allergy 2013; 43: 1189-95.

18. Bird JA, Leonard S, Groetch M, et al. Conducting an oral food challenge: an update to the 2009 adverse reactions to Foods Committee Work Group Report. J Allergy Clin Immunol Pract 2020; 8: 75-90.e17.

19. Lemon-Mule H, Sampson HA, Sicherer SH, et al. Immunologic changes in children with egg allergy ingesting extensively heated egg. I Allergy Clin Immunol 2008; 122: 977-83.e1.

20. Leonard SA, Sampson HA, Sicherer SH, et al. Dietary baked egg accelerates resolution of egg allergy in children. J Allergy Clin Immunol 2012; 130: 473-80.e1.

21. Sampson HA, Gerth van Wijk R, Bindslev-Jensen C, et al. Standardizing double-blind, placebo-controlled oral food challenges: American Academy of Allergy, Asthma \& Immunology-European Academy of Allergy and Clinical Immunology PRACTALL consensus report. J Allergy Clin Immunol 2012; 130: 1260-74.

22. Petrus NC, Schoemaker AF, van Hoek MW, et al. Remaining symptoms in half the children treated for milk allergy. Eur J Pediatr 2015; 174: 759-65.

23. Niggemann B. When is an oral food challenge positive? Allergy 2010; 65: 2-6.

24. Niggemann B, Beyer K. Pitfalls in double-blind, placebo-controlled oral food challenges. Allergy 2007; 62: 729-32.

25. Yanagida N, Minoura T, Kitaoka S, Ebisawa M. A three-level stepwise oral food challenge for egg, milk, and wheat allergy. J Allergy Clin Immunol Pract 2018; 6: 658-60.

26. Caffarelli C, Petroccione T. False-negative food challenges in children with suspected food allergy. Lancet 2001; 358: 1871-2.

27. Koplin JJ, Tang ML, Martin PE, et al. Predetermined challenge eligibility and cessation criteria for oral food challenges in the Health Nuts population-based study of infants. J Allergy Clin Immunol 2012; 129: 1145-7.

28. Niggemann B, Lange L, Finger A, et al. Accurate oral food challenge requires a cumulative dose on a subsequent day. J Allergy Clin Immunol 2012; 130: 261-3.

29. Chang A, Robison R, Cai M, et al. Natural history of foodtriggered atopic dermatitis and development of immediate reactions in children. J Allergy Clin Immunol Pract 2016; 4: 229-36.e1.

30. Eigenmann PA, Caubet JC, Zamora SA. Continuing foodavoidance diets after negative food challenges. Pediatr Allergy Immunol 2006; 17: 601-5. 